revascularization events. From 0-1 years, the cumulative incidence of TVRE was $15.9 \%$ and of NTVRE was $11.2 \%$. From 1-10 years, the cumulative incidences of TVRE and NTVRE were $12.3 \%$ and $22.4 \%$ respectively.

Conclusions At 10-years post PCI with new generation drug eluting stents, events related to remote vessel disease progression account for a higher proportion of events than events related to the stented vessel.

\section{AUTOMATED HIGH DENSITY LEFT ATRIAL VOLTAGE MAPPING OF LOW VOLTAGE AREAS IN ATRIAL FIBRILLATION DIFFERS FROM SINUS RHYTHM}

J Mannion, SJ Lennon, J Keaney, J Galvin, J O'Brien, G Jauvert, E Keelan, G Szeplaki, U Boles. Heart and Vascular Centre, Atrial Fibrillation and Cardiovascular Research Institute (CVRI), Mater Private Hospital Dublin, Ireland

\subsection{6/heartjin-2021-ICS.10}

Introduction Low voltage areas (LVAs) are used as surrogate markers for diseased left atrial (LA) tissue and scar distribution. LVAs can be overestimated when measured in atrial fibrillation (AF) due to factors such as variable contact between mapping catheter electrodes and the endocardium in the presence of multiple depolarisation wavefronts with typically decreased cycle lengths. Scar or substrate modification was previously recommended for those with persistent AF (PeAF). Correlation between voltages in sinus rhythm (SR) and AF have previously been measured in studies via point-bypoint analysis. An automated voltage histogram analysis (VHA) software module (CARTO3, Biosense Webster) has recently been validated for rapid area assessment of LA voltage maps and allows for very high-density analysis. We sought to compare LA LVA burden in SR to AF using VHA.

Methods We included patients over age 18 with PeAF who underwent de novo pulmonary vein isolation (PVI) with no additional lines, maps with $\geq 1000$ voltage points in each rhythm and a uniform procedure with initial mapping in AF then remapping in SR after PVI. We created 6 anatomical segments (AS) from each atrium for comparison in AF and SR
Abstract 10 Table 1 Correlation between LVAs identified in AF and SR

Table 1: Correlation values of measured LVAs in SR and AF via Spearman's correlation test of each anatomical segment. We have compared rhythms in either the Dense Scar $(\leq 0.2 \mathrm{mV})$ category or Diseased LA Tissue (0.21-0.5mV) category. *Denotes significance. $\mathrm{AS}=$ Anatomical segment, $\mathrm{AF}=$ Atrial fibrillation; $\mathrm{SR}=$ Sinus rhythm.

\begin{tabular}{lllll}
\hline AS & $\begin{array}{l}\text { Correlation } \\
\text { Coefficient, } \\
\text { AF and SR } \leq 0.2 \mathrm{mV}\end{array}$ & P value & $\begin{array}{l}\text { Correlation } \\
\text { Coefficient, } \\
\text { AF and SR 0.21-0.5 } \\
\mathrm{mV}\end{array}$ & P value \\
\hline Roof & 0.514 & & & \\
Lateral & 0.395 & $0.001^{*}$ & 0.152 & 0.247 \\
Septal & 0.425 & $0.012^{*}$ & 0.165 & 0.209 \\
Floor & 0.431 & $0.006^{*}$ & 0.252 & 0.052 \\
Posterior & 0.555 & $0.005^{*}$ & 0.091 & 0.49 \\
Anterior & 0.499 & $<0.001^{*}$ & 0.314 & $0.015^{*}$ \\
\hline
\end{tabular}

including the Anterior, Posterior, Roof, Floor, Lateral and Septal AS (figure 1). We removed the mitral annulus, trans-septal puncture site and pulmonary veins for VHA analysis. The total area of each AS was categorised by voltage into 10 aliquots between $0-0.5 \mathrm{mV}$, designating voltages $\leq 0.2 \mathrm{mV}$ as 'Dense Scar' and voltages $0.21-0.5 \mathrm{mV}$ as 'Diseased Tissue'. Data attained from VHA outputs were analysed using SPSS v.26.

Results Using VHA we analysed 58,342 ( $\mathrm{n}=10$ patients) voltage points between all AS $(\mathrm{N}=120)$. While comparing corresponding AS in voltage ranges consistent with Dense Scar, $\leq 0.2 \mathrm{mV}$, we found comparable LVA burden between rhythms in each AS except for the posterior wall, where there was significantly greater burden in AF $\left(159.03 \mathrm{~mm}^{2} \pm 194.65\right.$ Vs. $\left.102.14 \mathrm{~mm}^{2} \pm 157.47, \mathrm{p}=0.02\right)$. Utilising Spearman's correlation test (table 1) for voltages $\leq 0.2 \mathrm{mV}$ we found significant correlation coefficients in the range of $\mathrm{R}=0.395-0.555$ for each segment as displayed in figure 2. Assessing ranges consistent with Diseased Tissue, $0.21-0.5 \mathrm{mV}$, we found greater LVA burden in all AS when measured in AF, and this reached
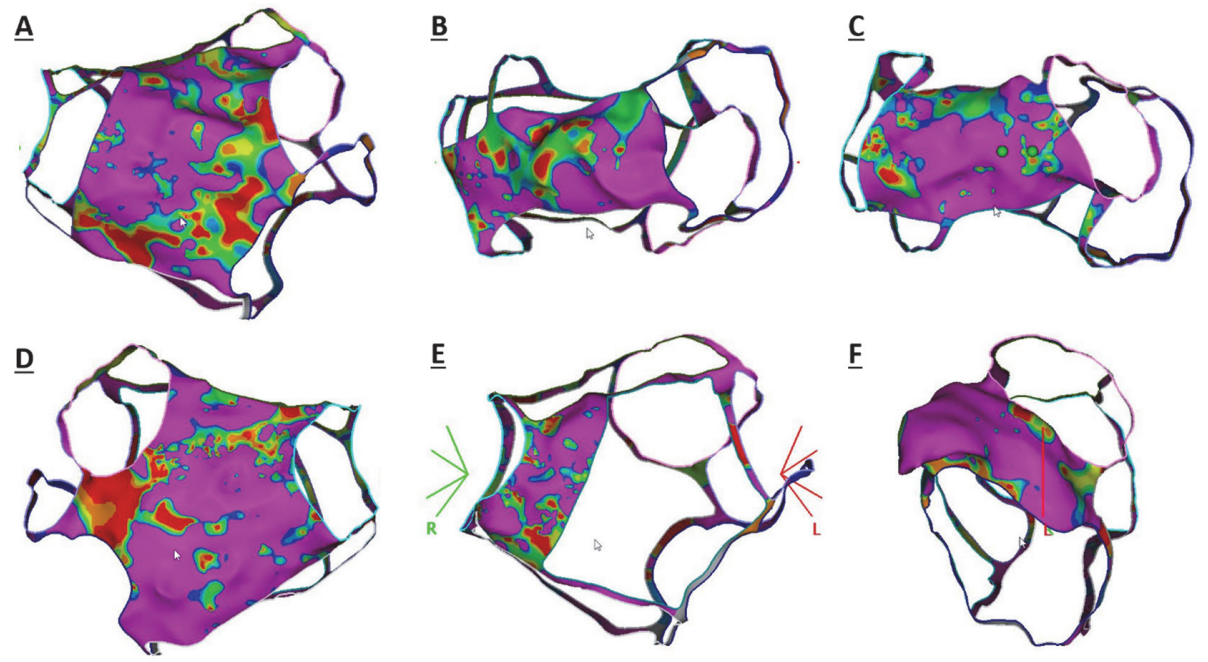

Abstract 10 Figure 1 Example of walls isolated from each LA

Figure 1: Demonstration of left atrial Anatomical Segments. A: Anterior. B: Roof. C: Floor. D: Posterior. E: Septal. F: Lateral. 


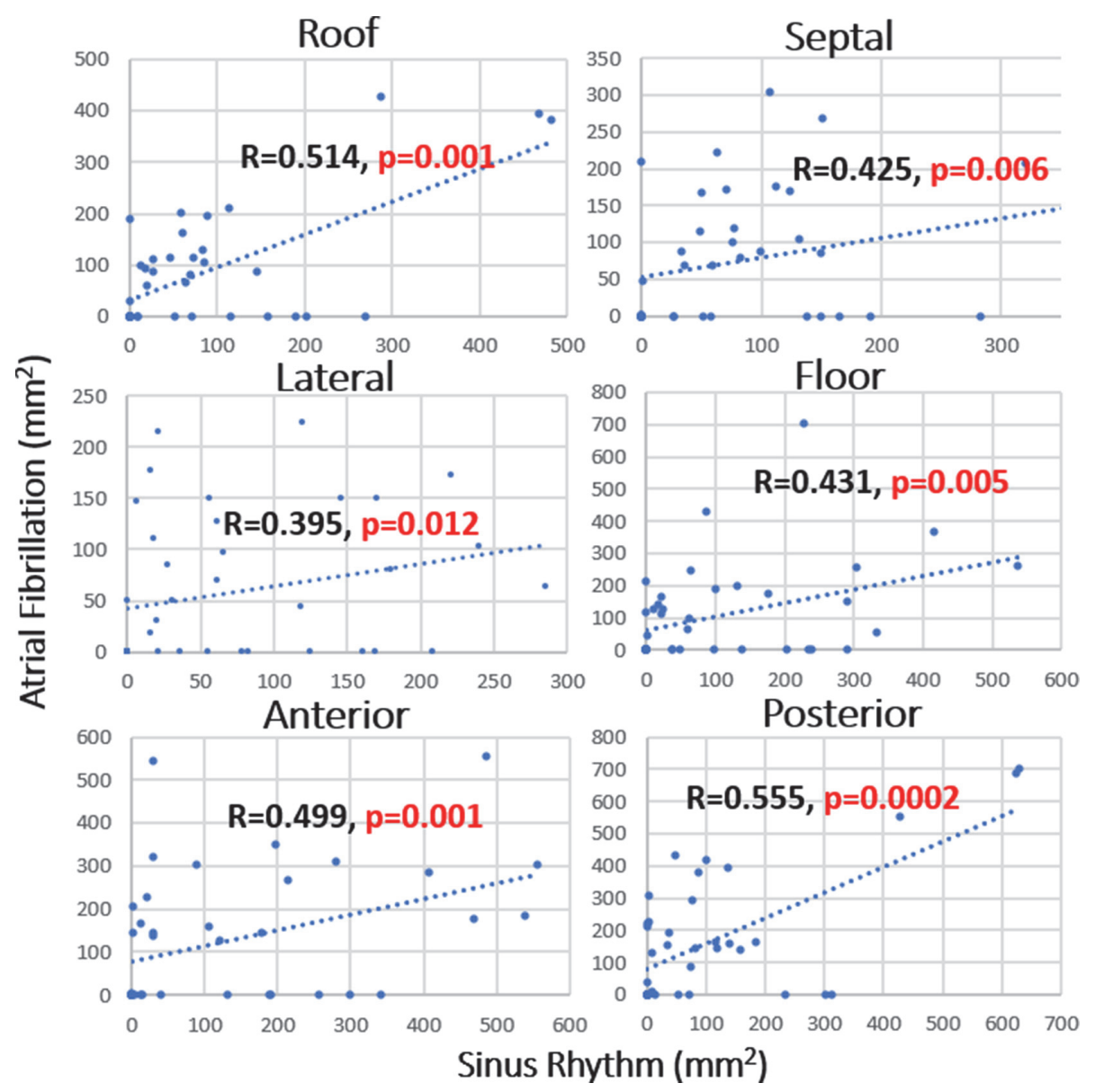

Abstract 10 Figure 2 Correlation scatterplot of LVAs $\leq 0.2 \mathrm{mV}$

Figure 2: Correlation coefficients of anatomical segment at voltages consistent with dense scar $\leq 0.2 \mathrm{mV}$ (in mm ${ }^{2}$ of endocardium fitting that voltage category) between rhythms of SR and AF.

significance in the Posterior $\left(\mathrm{AF}=234 \mathrm{~mm}^{2} \pm 150.45\right.$; SR $\left.=138.27 \mathrm{~mm}^{2} \pm 112.3, \mathrm{p}=<0.001\right)$, Roof $(\mathrm{AF}=150.61$ $\left.\mathrm{mm}^{2} \pm 93.17 ; \mathrm{SR}=115 \mathrm{~mm}^{2} \pm 77.14, \mathrm{p}=0.01\right)$, Anterior $\left(\mathrm{AF}=220.87 \mathrm{~mm}^{2} \pm 173.07 ; \mathrm{SR}=158.53 \mathrm{~mm}^{2} \pm 99.22\right.$ $\mathrm{p}=0.002)$ and Lateral AS $\left(\mathrm{AF}=137.05 \mathrm{~mm}^{2} \pm 104.99\right.$; $\left.\mathrm{SR}=87.52 \mathrm{~mm}^{2} \pm 66.82, \mathrm{p}=<0.001\right)$ as seen in table 1 . The voltage range of $0.21-0.5 \mathrm{mV}$ demonstrated weak correlation coefficients of $\mathrm{R}=0.09-0.31$, only reaching significance on the Posterior AS, once again shown in table 1.

Conclusions LVA burden $0.21-0.5 \mathrm{mV}$ in AF is significantly higher in SR with poor correlation, suggesting a greater influence of rhythm in this range and so care must be taken in interpretation. Comparatively, at dense scar voltages $(\leq 0.2$ $\mathrm{mV}$ ), we found comparable burden with good correlation between $\mathrm{AF}$ and SR, reflecting true readings in both rhythms. These findings should be carefully considered by operators performing additional scar modification and ablation.

\section{CLINICAL ASSOCIATIONS AND PATHOLOGICAL MECHANISMS OF REDUCED ATRIAL CONDUCTION VELOCITY}

D O'Hare, L O'Neill, I Sim, I Kotadia, R Bendikas, C Roney, S Niederer, JA Solis Lemus, O Razhegi, SE Williams, M O'Neill. King's College London, UK

10.1136/heartjn--2021-ICS.11
Conflict of Interest Dr. O'Hare has been the recipient of educational grants from Daiichi Sankyo and Johnson and Johnson Medical Ltd. during the course of this study.

Background The complex pathogenesis of atrial fibrillation (AF) remains unclear. A key concept is that for AF to be sustained, both a trigger and an atrial substrate capable of perpetuating the arrhythmia are required. Pathological remodelling in the atria can be observed after only a few minutes of high atrial rate, with the chronicity of these changes being directly related to the duration of the episodes of high atrial rate. This underpins the often-quoted axiom 'AF begets $\mathrm{AF}$ '. Increased atrial fibrosis in patients with $\mathrm{AF}$ has been observed in both histological and non-invasive studies using LGE-CMR atrial imaging. Reduced atrial conduction velocity $(\mathrm{CV})$ has been observed in fibrotic areas, which may propagate $\mathrm{AF}$ as more wavefronts can be sustained in the atria. It is unclear if reduced atrial $\mathrm{CV}$ is a prerequisite for $\mathrm{AF}$, or if it develops after the initial periods of arrhythmia. The use of 3D electro-anatomical mapping (EAM) has led to advancement in the accuracy of intra-cardiac CV measurement and investigations into the mechanism for reduced CV.

Hypothesis We hypothesised that left atrial CV plays a key role in the AF disease process and investigation into atrial $\mathrm{CV}$ may improve understanding into the pathogenesis and clinical course of AF. METHODS A systematic review was performed to assess current published evidence. This informed the design of a retrospective study to calculate $\mathrm{CV}$ from the left atrial 